\title{
Determination of Flavin Potential in Proteins by Xanthine/Xanthine Oxidase Method Elena Maklashina ${ }^{1,2, *}$ and Gary Cecchini ${ }^{1,2, *}$
}

\author{
${ }^{1}$ Molecular Biology Division, San Francisco VA Health Care System, San Francisco, CA 94121, USA; \\ ${ }^{2}$ Department of Biochemistry \& Biophysics, University of California, San Francisco, Ca 95158, USA \\ *For correspondence: Elena Maklashina@ucsf.edu; Gary.Cecchini@ucsf.edu
}

\begin{abstract}
[Abstract] This protocol describes a simple xanthine/xanthine oxidase enzymatic equilibration method for determination of the redox potential of a flavin. As an example of the use of this method, we determine the reduction potential of the covalently bound FAD cofactor $\left(E_{m}=-55 \mathrm{mV}\right)$ in the SdhA flavoprotein subunit of succinate dehydrogenase from Escherichia coli. In principle, this method can be used routinely to determine the redox potential of flavin cofactors in any simple flavoprotein from equilibrium concentrations with an appropriate reference dye of known $E_{m}$ without the use of sophisticated electrochemical equipment.
\end{abstract}

Keywords: Flavin, Flavoprotein, Reduction potential, Xanthine oxidase, Midpoint potential

[Background] Several biophysical methods can be used to measure the reduction midpoint potential $\left(E_{\mathrm{m}}\right)$ of flavins in a protein. These potentiometric methods usually rely on the electrochemical coupling between the protein of interest and an electrode. For example, in complex flavoproteins harboring additional cofactors such as iron-sulfur centers and quinones, electrochemical methods and Electron Paramagnetic Resonance (EPR) spectroscopy are often used to determine the $E_{m}$ of the redox centers (Kowal et al., 1995; Saenger, et al., 2005; Hudson et al., 2005; Cheng et al., 2015). However, in simple flavoproteins that contain only flavin redox centers, the co-factor can be directly studied by conventional optical spectrophotometry which does not require special electrochemistry equipment or expensive EPR instrumentation. In 1990 Vincent Massey introduced a simple method that allows determination of the flavin reduction potential from the equilibrium concentrations of the oxidized and reduced partners, i.e., flavoprotein and a reference dye (Massey, 1991). The method does not directly measure the reduction potential of the flavin but rather determines a difference between $E_{\mathrm{m}}$ values of the flavin and a reference dye. The scheme in Figure 1 describes the method. The xanthine/xanthine oxidase system provides a slow continuous reduction of the indicator dye and the flavin in the presence of benzyl viologen (BV) or methyl viologen (MV) which ensures the rapid equilibration of reducing equivalents. This allows slow changes in equilibrium of the reduced and oxidized forms of the protein and dye until both are completely reduced. A series of spectra recorded over the course of the reaction is used to calculate the ratio of the oxidized and reduced forms of flavin and dye. The Nernst plot of an equilibrium concentration, the dye against the flavoprotein allows determination of the shift in the $E_{m}$ of the protein in comparison with the dye. Because some of the components used in this method are low potential chemicals and proteins (viologen, reference dyes, and many flavoproteins) that are readily oxidized by even trace amounts of oxygen, a requirement for this system is that strict anaerobiosis be maintained. 
Here we show an example of how this method is applied to determine the reduction potential of covalently bound FAD in the SdhA flavoprotein of succinate dehydrogenase. SdhA is the flavoprotein component of the four subunit membrane-bound succinate:ubiquinone reductase (i.e., complex II) which is part of the TCA (Krebs) cycle and electron transport chain of the mitochondrion and many bacteria. Complex II couples the reaction of succinate oxidation to fumarate with ubiquinone reduction to ubiquinol. The FAD co-factor is involved in the reversible succinate-fumarate conversion. The $E_{\mathrm{m}}$ for free FAD in solution is $-219 \mathrm{mV}$, whereas covalent attachment of FAD to SdhA considerably raises the potential of the flavin. In this example, we use the redox dye indigo-tetrasulphonate (ITS) $\left(E_{\mathrm{m}}=-46 \mathrm{mV}\right.$ ) and have determined that the $E_{\mathrm{m}}{ }^{\mathrm{FAD}}$ for the Escherichia coli SdhA protein is $-55 \mathrm{mV}$. In principle, this simple method can be used for any flavoprotein by using a redox dye with a suitable reduction potential (i.e., a potential within $\pm 30 \mathrm{mV}$ to that expected for the flavoprotein being determined). This method has also been validated for the determination of the reduction potential of many other flavoproteins (see Christgen et al., 2019) and several heme proteins that suggests that the method could be widely useful for most heme proteins (Efimov et al., 2014).

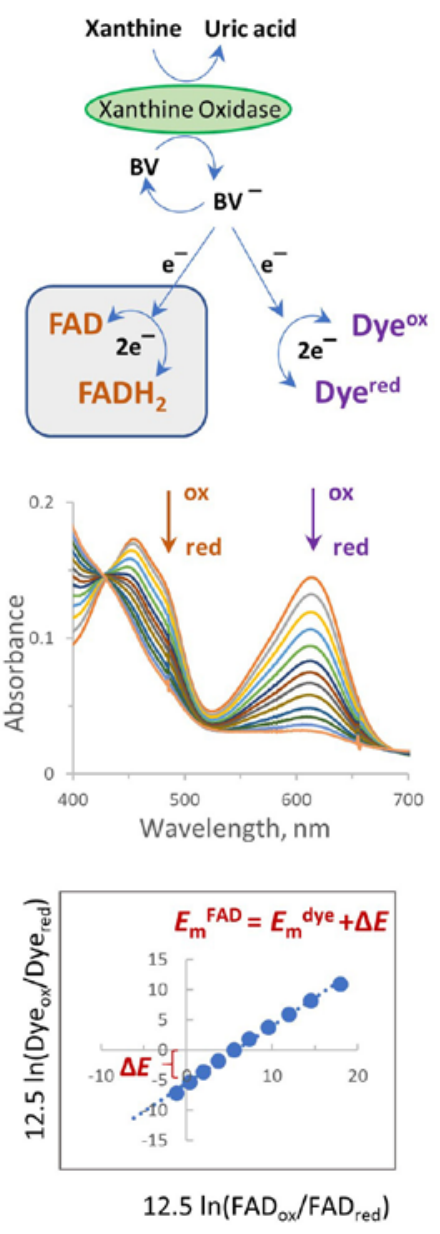

Figure 1. Spectrophotometric method for determination of the $E_{m}$ of a flavin using a reference dye in the presence of xanthine/xanthine oxidase. Most suitable dyes undergo 2-electron reduction (see Table 1). 


\section{Materials and Reagents}

1. $0.2 \mu \mathrm{m}$ filter (Corning) (Sigma-Aldrich, catalog number: CLS431229)

2. Potassium phosphate dibasic, anhydrous (Sigma-Aldrich, catalog number: 795496)

3. Potassium phosphate monobasic, anhydrous (Sigma-Aldrich, catalog number: 795488)

4. Xanthine (Sigma-Aldrich, catalog number: X7375)

5. Xanthine oxidase, lyophilized powder (Sigma-Aldrich, catalog number: X4376)

6. E. coli SdhA

E. coli SdhA was isolated with an N-terminally fused His-tag as described in Maklashina et al., 2018.

7. Glucose (Sigma-Aldrich, catalog number: G8270)

8. Catalase (Sigma-Aldrich, catalog number: C30)

9. Glucose oxidase (Sigma-Aldrich, catalog number: G2133)

10. Potassium indigotetrasulfonate (ITS) (Sigma-Aldrich, catalog number: 340596)

11. Benzyl viologen (Sigma-Aldrich, catalog number: 271845) or Methyl Viologen (Sigma-Aldrich, catalog number: 856177)

12. Flavoprotein, isolated SdhA (see the expression and purification method; Maklashina et al., 2018)

13. Ultra High Purity 5.0 Grade Argon (Airgas, catalog number: AR UHP300) or Ultra High Purity 5.0 Grade Nitrogen (Airgas, catalog number: NI UHP300)

14. Sodium Dithionite (Sigma-Aldrich, catalog number: 71699)

15. Apiezon High Vacuum grease type L (Fisher Scientific, catalog number: 50-365-186)

16. Nitrogen/argon, gas

17. Toluylene blue

18. Phenazine methosulfate

19. Thionine

20. Methylene blue

21. Pyocyanin

22. Indigotetrasulfonate

23. Resorufin

24. Indigotrisulfonate

25. Indigodisulfonate

26. 2-Hydroxy-1,4 naphthoquinone

27. Cresyl violet

28. Anthraquinone-2,6-disulphonate

29. Anthraquinone-2-sulfonate

30. Phenosafranine

31. Safranine $T$

32. $50 \mathrm{mM}$ potassium phosphate, $\mathrm{pH} 7.0$ (see Recipes) 
33. Xanthine solution, $10 \mathrm{mM}$ (see Recipes)

34. Xanthine oxidase solution (see Recipes)

35. Glucose solution, $1 \mathrm{M}$ (see Recipes)

36. Catalase solution (see Recipes)

37. Glucose oxidase solution (see Recipes)

38. Reference dyes solutions (see Recipes)

39. Benzyl viologen or methyl viologen (see Recipes)

\section{Equipment}

1. Spectrophotometer (for example, Agilent 8453 UV-visible spectrophotometer capable of scanning between 300 and $800 \mathrm{~nm}$, with a spectral resolution of $1 \mathrm{~nm}$ )

Temperature control is supplied by a Peltier unit from Agilent. Any means to maintain a constant temperature is appropriate (i.e., cuvette holder where the temperature is controlled by a water bath, for example).

2. An appropriate cuvette to conduct the reaction under strict anaerobic conditions (Figure 2)

\section{Considerations before the reaction:}

Choosing an anaerobic cuvette. This method relies on strict anaerobic conditions required for the duration of the reaction. Some labs may be well equipped for this type of study and have a spectrophotometer placed in an anaerobic hood. For many labs, however, this option may not be available, thus the reaction should be well isolated from an aerobic environment by using specialized optical cuvettes (Figure 2). The classical anaerobic cuvette (Figure 2A) has a side arm and air is exchanged by repeated cycles of applying a vacuum and replacing air with nitrogen/argon gas. The xanthine oxidase $(\mathrm{XO})$ which is used to initiate the reaction is placed in the side arm and mixed into the reaction after the cuvette is closed and sealed using vacuum grease. Alternatively, if the specialized cuvette shown in Figure $2 \mathrm{~A}$ is unavailable, a standard stoppered cuvette (Figure 2B) can be used. Additions are made by a gas tight syringe through an oxygen impermeable septum (Figure 2B, top). The most convenient method which was used by us and others is to use a common stoppered cuvette (Figure $2 \mathrm{~B}$, bottom). To achieve anaerobic conditions required for the assay, we use the glucose/glucose oxidase/catalase oxygen scavenging system that efficiently eliminates dissolved oxygen. In addition, the cuvette head space is flushed with a low flow of argon/nitrogen. To initiate the reaction, XO is added with an air tight syringe and after the injection the reaction is carefully mixed with the syringe needle. For some assays when using very low potential flavoproteins or dyes (i.e., a potential less than $-300 \mathrm{mV}$ ) the argon or nitrogen gas should be additionally treated by an oxygen scrubbing system to remove traces of oxygen. The use of an air tight cuvette (Figure 2A) would be also beneficial. The final volume of the reaction is usually $1 \mathrm{ml}$ but can be scaled up or down depending on the cuvette type and amount of protein to be studied. 


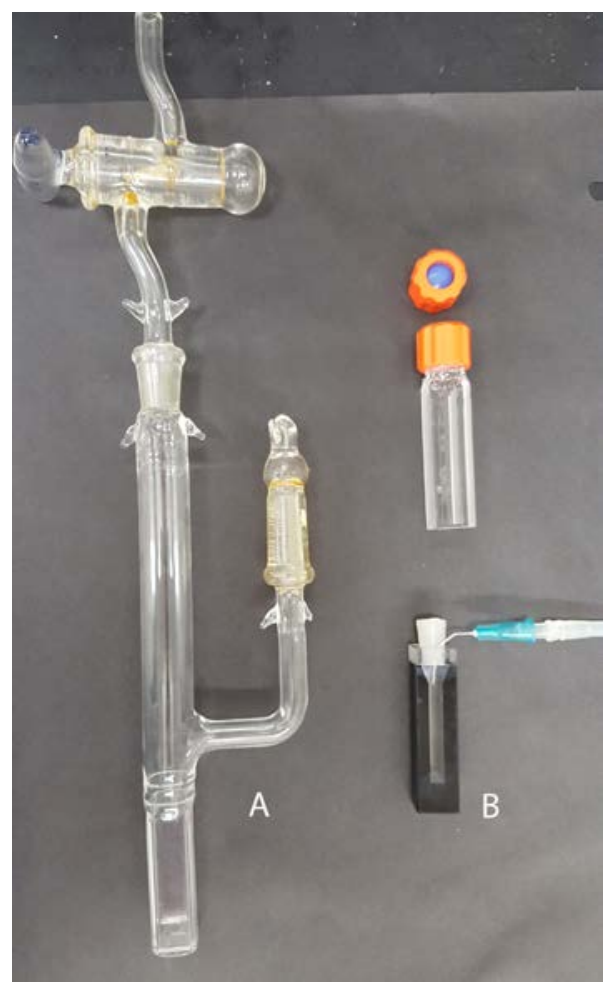

Figure 2. Optical cuvettes that can be used for anaerobic measurements. A. A sealed anaerobic cuvette with a side arm. B. A cuvette with a septum (top) and a standard optical cuvette equipped with a silicone stopper (bottom). Both cuvettes can be used with a gas line to supply argon/nitrogen (shown for the bottom cuvette).

Choosing an appropriate dye. As a general rule, the $E_{m}$ of a reference dye should be within $\pm 30 \mathrm{mV}$ of the anticipated flavin potential in the protein of interest. Table 1 summarized some reference dyes that had been used for determination of flavin reduction potentials. Some tips for choosing a dye: for mutants of a flavoprotein with known potential start with a dye which has an $E_{m}$ closest to the wild-type. For example, a mutation may result in a change of flavin attachment to the protein. Covalently bound flavins demonstrate higher reduction potentials than non-covalent flavins (Heuts et al., 2009). Since wild-type SdhA has a flavin potential of about $-50 \mathrm{mV}$, we used a dye (ITS) which has a redox potential near this value (see Table 1). 
Table 1. Reference Dyes

\begin{tabular}{cc}
\hline Dye & $E_{m}(\mathrm{mV})$ \\
\hline Toluylene blue & +115 \\
Phenazine methosulfate & +80 \\
Thionine & +64 \\
Methylene blue & +11 \\
Pyocyanin & -34 \\
Indigotetrasulfonate & -46 \\
Resorufin & -79 \\
Indigotrisulfonate & -81 \\
Indigodisulfonate & -125 \\
2-Hydroxy-1,4 naphthoquinone & -142 \\
Cresyl violet & -166 \\
Anthraquinone-2,6-disulphonate & -184 \\
Anthraquinone-2-sulfonate & -225 \\
Phenosafranine & -252 \\
Safranine T & -289 \\
Benzyl viologen* & -359 \\
Methyl viologen* & -446 \\
\hline
\end{tabular}

*Viologens are one-electron reduction dyes, all others undergo two-electron reduction.

Choice of the wavelength to monitor absorbance changes in the flavin and dye. As depicted in Figures 1 and 4, the oxidized and reduced forms of the flavin and dye are calculated from the spectra containing both components. Thus, wavelengths used for the analysis should represent where spectral changes are attributed to only one component and where absorbance changes contributed by the second component are negligible. For example, the maximum absorbance of FAD in SdhA is around $460 \mathrm{~nm}$, however, the reduced form of ITS also absorbs at this wavelength and will interfere with correct determination of FAD concentrations. A separate spectrum of dithionite reduced ITS, however, shows that $485 \mathrm{~nm}$ is the isosbestic point for ITS (demonstrated in Figure 3). FAD also retains significant absorbance at $485 \mathrm{~nm}$, thus this wavelength can be used for calculations of reduced/oxidized forms of FAD without any contributions from ITS. Reduction of ITS was measured at $614 \mathrm{~nm}$, a peak absorbance, where there is no interference from flavin absorption. Thus, the wavelengths of $485 \mathrm{~nm}$ for the flavin and $614 \mathrm{~nm}$ for the dye can be used for the analysis. 


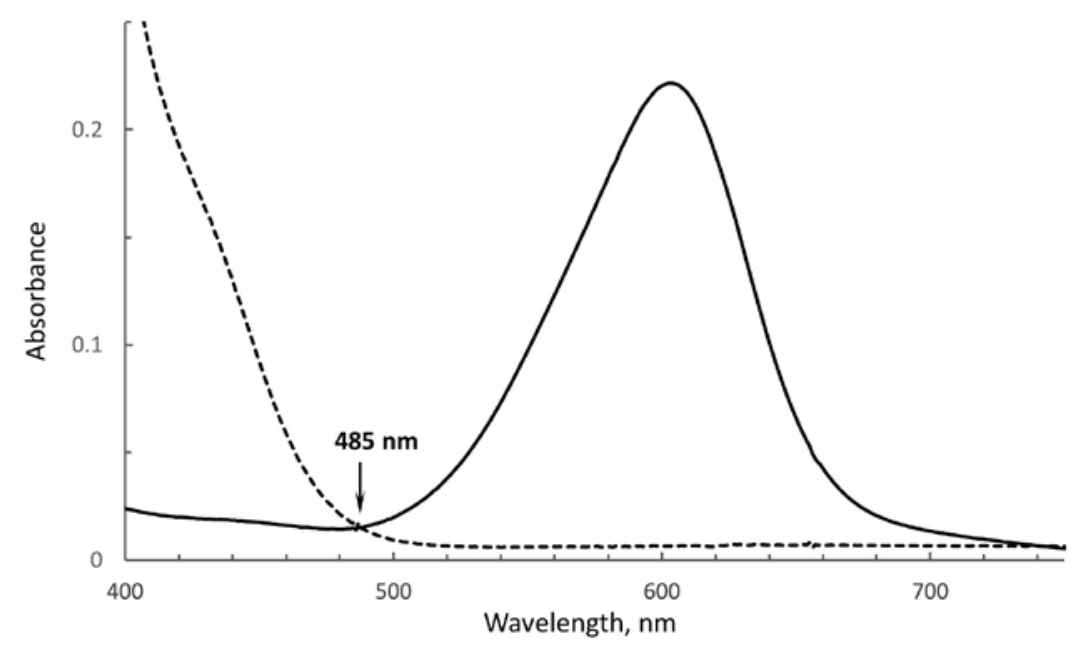

Figure 3. The isobestic point for ITS. The spectrum of oxidized ITS is shown as the solid line and the dithionite reduced ITS is shown as the dashed line. Both forms show the same absorbance at $485 \mathrm{~nm}$, i.e., the isobestic point.

3. An air tight syringe for making additions to the anaerobic assay

\section{$\underline{\text { Software }}$}

1. Microsoft Excel or any other spread sheet program for data analysis

\section{Procedure}

1. For this assay, we utilized the conventional optical cuvette with argon shown in Figure 2B (bottom). In the cuvette add $50 \mathrm{mM}$ potassium phosphate buffer $\left(\mathrm{pH} 7.0,25^{\circ} \mathrm{C}\right)$, and reagents to indicated final concentration: $10 \mathrm{mM}$ glucose, $5 \mu \mathrm{g} / \mathrm{ml}$ catalase, $0.3 \mathrm{mM}$ xanthine, $50 \mu \mathrm{g} / \mathrm{ml}$ glucose oxidase, and benzyl viologen (10-20 $\mu \mathrm{M})$. A low flow of argon/nitrogen is layered on the top of the cuvette (no bubbling). Record the baseline spectra.

2. Add sufficient flavoprotein (SdhA) to give an absorbance of 0.1-0.5 at 450-460 nm, which corresponds to 8-40 $\mu \mathrm{M}$ FAD (mix carefully). The maximum absorbance should be similar between the FAD at $\sim 460 \mathrm{~nm}$ and the dye (ITS at $614 \mathrm{~nm}$ ). The spectrum is scanned between 350 and $750 \mathrm{~nm}$, and recorded. This spectrum corresponds to the oxidized states of the flavoprotein and the dye.

3. Wait about 15 min to establish anaerobic conditions and then add xanthine oxidase in an air tight syringe (10-50 $\mathrm{nM}$ is the usual range). The concentration of xanthine oxidase should be adjusted so that electron transfer is sufficiently slow so that the overall system remains in equilibrium at all times. Set the spectrophotometer to record spectra with 1-3 min intervals. The concentration of reagents should be such that complete reduction of the flavin and dye occur within 1-4 h. 
4. The reaction is stopped when spectra show no further changes or the benzyl viologen begins to be reduced as indicated by an increase in absorbance in the 550-600 $\mathrm{nm}$ region.

5. Finally, transfer the individual spectra (wavelength vs absorbance) from the spectrophotometer and combine them in an Excel spreadsheet. Figure 4 demonstrates the set of spectra of a single experiment for the reduction of SdhA with ITS.

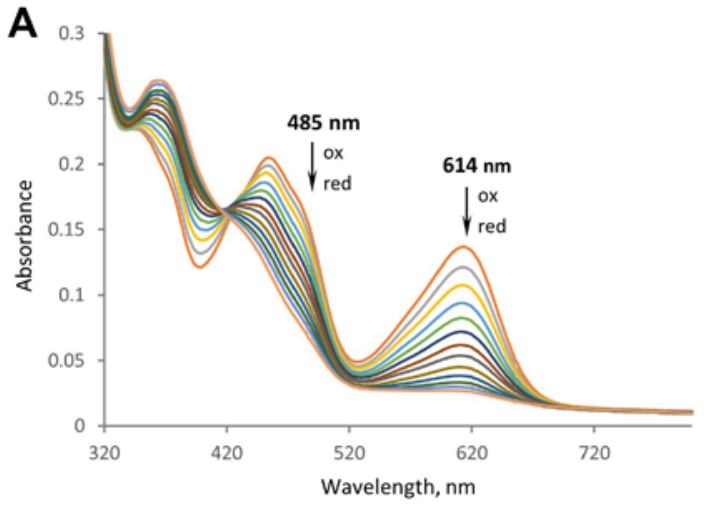

B

\begin{tabular}{|r|r|r|r|r|r|}
\hline \multicolumn{2}{|l|}{ Absorbance (AU) } & & & \\
\hline & \multicolumn{1}{|l|}{ Spec1 } & 2 & 3 & 4 & 5 \\
\hline $\begin{array}{l}\text { Wavelength } \\
\text { (nm) }\end{array}$ & & & & & \\
\hline 320 & 0.20668 & 0.266682 & 0.264405 & 0.265023 & 0.265848 \\
\hline 321 & 0.203111 & 0.259537 & 0.257677 & 0.258677 & 0.2595 \\
\hline 322 & 0.199914 & 0.253046 & 0.251618 & 0.252632 & 0.253474 \\
\hline 323 & 0.197504 & 0.247641 & 0.246331 & 0.247655 & 0.248822 \\
\hline 324 & 0.195053 & 0.242539 & 0.241475 & 0.242908 & 0.243943 \\
\hline 325 & 0.193345 & 0.238048 & 0.237378 & 0.239011 & 0.240015 \\
\hline 326 & 0.191977 & 0.234234 & 0.23369 & 0.235524 & 0.236671 \\
\hline
\end{tabular}

Figure 4. Optical changes observed during anaerobic reduction of SdhA and ITS in the presence of xanthine/xanthine oxidase. A. Graph combining individual spectra. B. An example of the Excel spreadsheet that combined 5 spectra taken during the measurement.

\section{Troubleshooting}

1. Major contributors for the incomplete reduction (or lack thereof) of the flavin and the dye are an oxygen leak and/or low xanthine oxidase activity. A suggested test to check the anaerobic conditions, i.e., the reduction of benzyl viologen by $X O$ is to omit the flavoprotein and dye from the assay. Reduction of $\mathrm{BV}\left(E_{m}=-359 \mathrm{mV}\right)$ should be observed under anaerobic conditions. The oxidized form of $\mathrm{BV}$ is colorless and a broad peak forms at $550-600 \mathrm{~nm}$ when $\mathrm{BV}$ begins to undergo reduction.

2. If the almost complete reduction of either the flavin or dye precedes the reduction of the second partner, then the difference in reduction potentials between the flavin and the dye has exceeded the requirements for this method and different dye should be chosen. The component with the higher potential will be reduced first and this will help to determine the reduction potential of the next dye to try.

\section{Data analysis}

The difference in $E_{m}$ potentials of the protein and dye, is calculated from a plot where the ratio of oxidized/reduced forms for the protein and the dye corresponding for each spectrum are plotted against each other.

For this calculation, the data is fitted to the Nernst plot. The step by step derivation is taken from Efimov et al. (2014). 
The reduction potentials of the flavin containing protein (P) and dye (D) are determined by using the Nernst equation:

$$
\begin{aligned}
& E_{P}=E_{m, P}+\frac{R T}{n F} \ln \left(\frac{\left[P_{o x}\right]}{\left[P_{\text {red }}\right]}\right) \\
& E_{D}=E_{m, D}+\frac{R T}{n F} \ln \left(\frac{\left[D_{o x}\right]}{\left[D_{r e d]}\right.}\right)
\end{aligned}
$$

Slow rates of electron input by $\mathrm{XO}$ ensure the equilibrium of the oxidized and reduced forms of the protein and dye at any given time point. Thus, their electrochemical potentials also equal:

$$
\begin{gathered}
E_{P}=E_{D} \\
E_{m, P}+\frac{R T}{n F} \ln \left(\frac{\left[P_{o x}\right]}{\left[P_{r e d}\right]}\right)=E_{m, D}+\frac{R T}{n F} \ln \left(\frac{\left.D_{o x}\right]}{\left.D_{r e d}\right]}\right)
\end{gathered}
$$

Defining $x$ as a Nernst concentration term for the protein, and $y$ as a Nernst concentration term for the dye.

$$
\begin{gathered}
x=\frac{R T}{n F} \ln \left(\frac{\left[P_{o x}\right]}{\left[P_{r e d}\right]}\right) \\
y=\frac{R T}{n F} \ln \left(\frac{\left[D_{o x}\right]}{\left[D_{r e d}\right]}\right) \\
E_{m, P}+x=E_{m, D}+y
\end{gathered}
$$

When the protein is in redox equilibrium $\left[P_{o x}\right]=\left[P_{r e d}\right], x=0 ; y$ is defined as $\Delta E$, the difference in mid-point potentials of the protein and the dye.

$$
E_{m, P}=E_{m, D}+\Delta E
$$

To determine $\Delta E$, the Nernst concentration term for the dye $(y)$ and the Nernst concentration term for the protein $(x)$ are calculated from each spectrum and plotted against each other. The ratio of [oxidized]/[reduced] concentrations for the protein and the dye is determined using the wavelengths designated for each component. In our experiment, we used $485 \mathrm{~nm}$ for FAD and $614 \mathrm{~nm}$ for ITS.

$$
\frac{\text { [oxidized] }}{\text { [reduced] }}=\frac{A-A_{\min }}{A_{\max }-A}
$$


where $A_{\max }$ is the absorbance of the oxidized forms, $A_{\min }$ is the absorbance of the reduced forms, and $A$ is the absorbance taken from each spectrum. Now using the values of the thermodynamic constants $\mathrm{RT} / \mathrm{nF}$ is equal to 12.5 ; $(R=$ the gas constant; $T$ = the temperature in Kelvin, $F=$ Faraday's constant; $n=$ number of electrons for FAD and ITS reduction, $n=2$ for both FAD and ITS) and obtained the final equations for $x$ and $y$.

$$
\begin{aligned}
& x=12.5 \ln \left(\frac{A-A_{\min }}{A_{\max }-A}\right) \\
& y=12.5 \ln \left(\frac{A-A_{\min }}{A_{\max }-A}\right)
\end{aligned}
$$

Below, we use the data from the set of spectra (Figure 5) to calculate $E_{m}{ }^{F A D}$ in SdhA.

\section{Calculations}

1. We determine $A_{\max }$ and $A_{\min }$ for the protein FAD (column B) and the dye (column D) (Figure 4) from the oxidized spectrum and from the spectrum at the end of the reaction.

2. Next, using absorbance at a chosen wavelength (columns $B$ and $D$ ) in each spectrum the Nernst concentrations terms for the protein FAD, (column C) and dye (column E) are calculated (Figure $5 A)$. The graphic representation of $y$ vs. $x$ gives a straight line with a slope of one and an intercept equal to $y$, or $\triangle E$, a shift in the midpoint potentials between the protein FAD and dye (Figure 5B). Thus, the $E_{m}{ }^{F A D}$ in SdhA is $-55.5 \mathrm{mV}$. This value is similar to the $E_{m}{ }^{F A D}$ determined for the four-subunit succinate dehydrogenase complex determined by potentiometric titration after correction for the different pH used for analysis (Cheng et al., 2015). Since the FAD is attached covalently in SdhA, the midpoint potential is significantly higher than the $E_{m}{ }^{F A D}$ of free flavin $(-219 \mathrm{mV})$. 


\begin{tabular}{|c|c|c|c|c|}
\hline A & B & c & D & $\mathrm{E}$ \\
\hline & FAD in SdhA & & ITS & \\
\hline & & & & \\
\hline & $\mathrm{A} 485 \mathrm{max}=0.135$ & $x=12.5 \ln ($ FADox/FADred) & A614max $=0.144$ & $y=12.5 \ln ($ ITSox $/$ ITSred $)$ \\
\hline & A485min $=0.064$ & & $A 614 \mathrm{~min}=0.026$ & \\
\hline & & & & \\
\hline & Absorbance & $12.5^{*} \ln ((\mathrm{Bn}-\mathrm{A} 485 \mathrm{~min}) /(\mathrm{A} 485 \mathrm{max}-\mathrm{Bn}))$ & Absorbance & $12.5^{*} \ln ((\mathrm{Dn}-\mathrm{A} 485 \mathrm{~min}) /(\mathrm{A} 485 \mathrm{max}-\mathrm{Dn}))$ \\
\hline & $485 \mathrm{~nm}$ & & $614 \mathrm{~nm}$ & \\
\hline Hspectrum & & & & \\
\hline & & & & \\
\hline 1 & 0.131 & 34.351 & 0.132 & 27.595 \\
\hline 2 & 0.124 & 21.556 & 0.119 & 16.550 \\
\hline 3 & 0.117 & 13.596 & 0.106 & 9.429 \\
\hline 4 & 0.110 & 7.493 & 0.094 & 4.046 \\
\hline 5 & 0.103 & 2.265 & 0.083 & -0.845 \\
\hline 6 & 0.098 & -0.967 & 0.075 & -4.297 \\
\hline 7 & 0.093 & -4.338 & 0.067 & -7.990 \\
\hline 8 & 0.088 & -8.623 & 0.058 & -12.455 \\
\hline 9 & 0.081 & -14.295 & 0.048 & -18.292 \\
\hline 10 & 0.077 & -19.076 & 0.042 & -23.583 \\
\hline 11 & 0.073 & -24.806 & 0.036 & -29.803 \\
\hline 12 & 0.069 & -31.695 & 0.032 & -36.952 \\
\hline
\end{tabular}

B

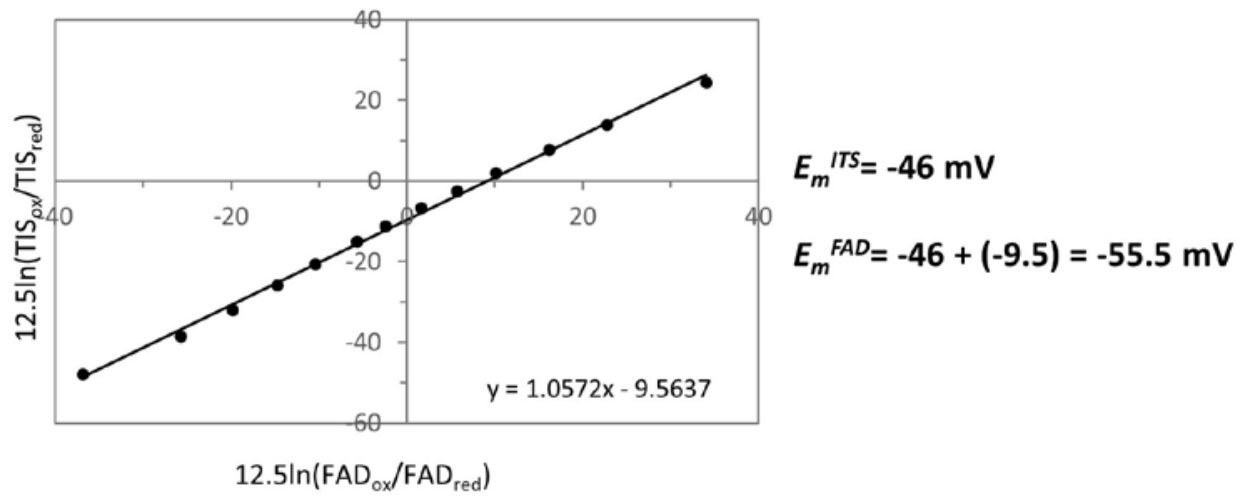

Figure 5. Final Calculations. A. An example of the Excel spreadsheet with calculations of the Nernst concentration terms for SdhA and ITS. B. The corresponding Nernst plot.

Notes:

a. It is often observed that the points at the far ends of the plot are out of alignment. This corresponds to the states of the reaction where the protein and dye are predominately in their oxidized and reduced forms, and equilibrium takes longer than the rate of the XO reaction allows. Therefore, these points are often omitted from the graph until the experimental points form a straight line with a slope equal to one.

$b$. This experiment demonstrates the $E_{m}$ determination of the $2 e^{-}$reduction of FAD/FADH $H_{2}$ in SdhA. In some flavoproteins 1e- reduction of FAD may produce a stable semiquinone radical FAD that can be observed as an intermediate form before reduction to hydroquinone.

\section{Recipes}

1. $50 \mathrm{mM}$ sodium phosphate $(\mathrm{pH} 7.0)$ 
Dissolve $4.67 \mathrm{~g}$ of $\mathrm{K}_{2} \mathrm{HPO}_{4}$ (dibasic potassium phosphate) and $3.15 \mathrm{~g}$ of $\mathrm{KH}_{2} \mathrm{PO}_{4}$ (monobasic potassium phosphate) and bring the final volume to 1-liter with distilled water

2. Xanthine solution, $10 \mathrm{mM}$

Dissolve $15.2 \mathrm{mg}$ of xanthine in $9.9 \mathrm{ml}$ of distilled water and $0.1 \mathrm{ml}$ of $1 \mathrm{M}$ sodium hydroxide (stored at $-20^{\circ} \mathrm{C}$ )

3. Xanthine oxidase solution

$0.2 \mathrm{mM}$ in $50 \mathrm{mM}$ potassium phosphate $\mathrm{pH} 7.0$ (stored at $-20^{\circ} \mathrm{C}$ )

4. Glucose solution, $1 \mathrm{M}$

$1 \mathrm{M}$ made in distilled water

For longer storage, filter sterilize using a $0.2 \mu \mathrm{m}$ filter (Corning) (stored at $-20^{\circ} \mathrm{C}$ )

5. Catalase solution

Catalase $(5 \mathrm{mg} / \mathrm{ml})$ in $50 \mathrm{mM}$ potassium phosphate $\mathrm{pH} 7.0$ (stored at $-20^{\circ} \mathrm{C}$ )

6. Glucose oxidase solution

Glucose oxidase $(20 \mathrm{mg} / \mathrm{ml})$ in $50 \mathrm{mM}$ potassium phosphate $\mathrm{pH} 7.5$ (stored at $-20^{\circ} \mathrm{C}$ )

7. Reference dyes solutions

10 to $20 \mathrm{mM}$ solutions in water (stored at $4{ }^{\circ} \mathrm{C}$ )

8. Benzyl viologen or methyl viologen

Benzyl viologen or methyl viologen to a final concentration of $10 \mathrm{mM}$ in water

\section{Acknowledgments}

Research in our laboratory was supported by the National Institutes of Health grant RO1 GM61606.

GC is also supported by the Department of Veterans Affairs Research Senior Career Scientist award \#1K6BX004215. This protocol was adapted from the method of Vincent Massey (Massey, 1991) as described in Maklashina et al. (2018) and the redox calculations were adapted from Efimov et al. (2014).

\section{Competing interests}

The authors declare no competing interests.

\section{References}

1. Cheng, V. W., Piragasam, R. S., Rothery, R. A., Maklashina, E., Cecchini, G. and Weiner, J. H. (2015). Redox state of flavin adenine dinucleotide drives substrate binding and product release in Escherichia coli succinate dehydrogenase. Biochemistry 54(4): 1043-1052.

2. Christgen, S. L., Becker, S. M. and Becker, D. F. (2019). Methods for determining the reduction potentials of flavin enzymes. Methods Enzymol 620: 1-25. 
3. Efimov, I., Parkin, G., Millett, E. S., Glenday, J., Chan, C. K., Weedon, H., Randhawa, H., Basran, J. and Raven, E. L. (2014). A simple method for the determination of reduction potentials in heme proteins. FEBS Lett 588(5): 701-704.

4. Heuts, D. P., Scrutton, N. S., McIntire, W. S. and Fraaije, M. W. (2009). What's in a covalent bond? On the role and formation of covalently bound flavin cofactors. FEBS J 276(13): 34053427.

5. Hudson, J. M., Heffron, K., Kotlyar, V., Sher, Y., Maklashina, E., Cecchini, G. and Armstrong, F. A. (2005). Electron transfer and catalytic control by the iron-sulfur clusters in a respiratory enzyme, E. coli fumarate reductase. J Am Chem Soc 127(19): 6977-6989.

6. Kowal, A. T., Werth, M. T., Manodori, A., Cecchini, G., Schröder, I., Gunsalus, R. P. and Johnson, M. K. (1995). Effect of cysteine to serine mutations on the properties of the [4Fe-4S] center in Escherichia coli fumarate reductase. Biochemistry 34(38): 12284-12293.

7. Maklashina, E., Rajagukguk, S., Iverson, T. M. and Cecchini, G. (2018). The unassembled flavoprotein subunits of human and bacterial complex II have impaired catalytic activity and generate only minor amounts of ROS. J Biol Chem 293(20): 7754-7765.

8. Massey, V. (1991). A simple method for the determination of redox potentials. In: Flavins and Flavoproteins 1990. Curti, B., Ronchi, S. and Zanetti, G. (Eds.) Walter de Gruyter \& Co., Berlin, pp. 59-66.

9. Saenger, A. K., Nguyen, T. V., Vockley, J. and Stankovich, M. T. (2005). Thermodynamic regulation of human short-chain acyl-CoA dehydrogenase by substrate and product binding. Biochemistry 44(49): 16043-16053. 